\title{
Routing for Mobile Ad-hoc Network towards Quality of Services
}

\author{
Manpreet Kaur \\ Research scholar \\ Lovely University, Phagwara
}

\author{
Jyoti Kohli \\ Assistant professor \\ Dept of computer science \\ Lovely University, Phagwara
}

\begin{abstract}
A mobile ad-hoc network is an autonomous network that can be formed without any established infrastructure, where nodes can move arbitrary in the topology with variation of speed and trajectory. Mobile Ad-hoc Networks prove to be the best fit for various applications like Military operations, Flood affected areas, Remote area etc. Ad-hoc Networks are limited with energy and generally more venerable to energy depletion due to routing and other running processes. Clustering in the mobile ad-hoc devices can save the precious energy of the devices. In our research we are using ant colony optimization for providing stability in clustering process in mobile ad-hoc network. Our target is to improve the performance of the overall mobile network.
\end{abstract}

Keywords: Mobile Ad-hoc Network, Ant colony Algorithm, Cluster, AODV.

\section{INTRODUCTION}

Ad-hoc networks is centralized networks, such as GSM, don't have capability to use everywhere in all situations. Some popular examples of these types of networks include establishing survivable monitoring, reliable with efficiency, dynamically adaptive communication for rescue operations in emergency, disaster relief efforts and different type of unique/ non-unique military networks. These types of network scenarios cannot be a centralized and organized connectivity and it can be act as applications of MANETs. The huge chunk of applications for MANETs is widely ranging from small to largely diversify, ranging from small to big, static to dynamic networks that are constrained by very limited power sources, mobility terms comes in scene always, and highly movable or dynamic networks.

To use or enable multi-hop communication in a widely driven and distributed environment, all nodes should be capable of acting as small transferring router/ switch according to demand (see Figure 1). Intermediate path (Routes) has been created and maintained by some routing protocol. MANET routing protocol designed in a complexity of having capability to handle the dynamically changing environment as discussed earlier so that rapidly changing topologies and environments can be covered by these protocols. For route maintenance one has two main approaches in MANETs, reactive and proactive. Reactive routing protocols create on demand routes which saves huge resources. On the other hand proactive protocols have to maintain whole table of topology in timely dynamic environment.

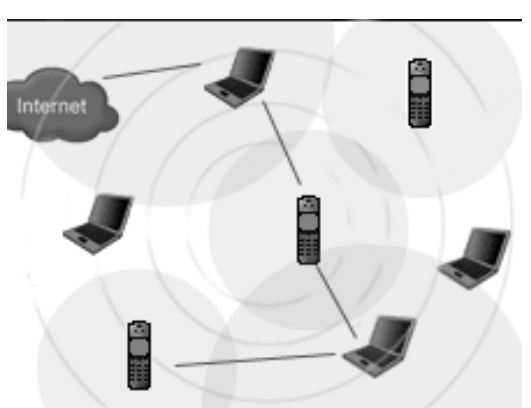

Figure 1. A typical MANET [4]

Mobility varies according to the network behavior and each node act as a router for next node for the communication. Traffic is only transmitted to nearby neighbors within the radio range when these network working in the ad-hoc manner, which in turns required manet routing protocols to set up and maintain traffic paths.

\section{ROUTING IN MANET}

Routing means to choose a path. Routing in MANET means to choose a right and suitable path from source to destination. Routing terminology is used in different kinds of networks such as in telephony technology, electronic data networks and in the internet network. Here work is more concern about routing in mobile ad hoc networks [4]. Routing protocols in mobile ad hoc network means that the mobile nodes will search for a route or path to connect to each other and share the data packets [2]. Protocols are the set of rules through which two or more devices (mobile nodes, computers or electronic devices) can communicate to each other. In mobile ad hoc networks the routing is mostly done with the help of routing tables. These tables are kept in the memory cache of these mobile nodes [4]. When routing process is going on, it route the data packets in different mechanisms. The first is uni-cast, in which the source directly sends the data packets to the destination. The sec is multicast, in this the source node sends data packet to the specified multiple nodes in the network [4]. The third is broadcast; it means the source node sends messages to all the near and far nodes in the network.

Routing has two basic types, which are as under.

Static Routing: is done by the administrator manually to forward the data packets in the network and it is permanent. No any administrator can change this setting [3]. These static routers are configured by the administrator, which means there is no need to make routing tables by the router itself. 
Dynamic Routing: is automatically done by the choice of router. It can route the traffic on any route depend on the routing table. Dynamic routing allows the routers to know about the networks and the interesting thing is to add this information in their routing tables. In dynamic routing the routers exchange the routing information if there is some change in the topology [4]. Exchanging information between these dynamic routers learn to know about the new routes and networks. Dynamic routing is more flexible than static routing. In dynamic routing it have the capability to overcome the overload traffic. Dynamic routing uses different paths to forward the data packets. Dynamic routing is better than static routing [4].

\section{AODV AND QUALITY OF SERVICES IN MANET}

AODV is an on-demand routing protocol. The AODV algorithm gives an easy way to get change in the link situation. For example if a link fails notifications are sent only to the affected nodes in the network. This notification cancels all the routes through this affected node. [6] The Quality of Service in MANET is imperative that we understand how Quality of Service is currently provided in wire-line networks. In the field of packet-switched networks and computer networking, the traffic engineering term Quality of Service ( QoS ) refers to the probability of the telecommunication network meeting a given traffic contract, or in many cases is used informally to refer to the probability of a packet succeeding in passing between two points in the network.

A traffic contract (SLA, Service Level Agreement) specifies the assurances for the ability of a network to give guaranteed performance/throughput/latency bounds based on mutually agreed measures, usually by prioritizing traffic. Broadly speaking there are two types of service which require QoS: (1) Elastic and (2) Inelastic. Inelastic service, meaning that they require a certain level of bandwidth to function - any more than required is unused, and any less will render the service non-functioning. [6] By contrast, elastic applications can take advantage of however much or little bandwidth is available. MANET is expected to provide QoS for Elastic services. In general QoS is the result of "coordinated effort" between various network entities. Such an effort initiative may be formally defined in the form of SLA between network entities in the case of wire-line networks. [8] For MANET the concept of QoS would result from "coordinated effort" between various nodes.

\section{PROBLEM DEFINATION}

Manet is a multi-hop ad-hoc network where nodes can move arbitrary in the topology with variation of speed and trajectory. The Manet network has no infrastructure or dependency and can be implement quickly in any environment but due to limited computing power, low bandwidth, high mobility and absence of central coordinating entity, behavior of different routing protocols are difficult to calculate in different environment. Networks suffer the problem of routing during link failure in between nodes or cluster of nodes. The protocol design presented the mantes in finding the best path or route for data communication. Since, Mantes are typically used as wireless backbones, and they have the nature that the wireless communication is not constant. Hence, it is important to define them against link or node failures. Better clustering approach is required to implement. In particularly Ant Colony has been implemented to provide stability in routing process of Mobile ad-hoc networks.

\section{IMPLIMENTATION}

To implement our concept of clustering and optimization of routing through Ant Colony method, we have used the below parameters given in Table 1.

\begin{tabular}{|c|c|}
\hline Parameter & Value \\
\hline Simulation Time & 4500 Seconds \\
\hline Number of Nodes & 50 \\
\hline Data Rate & $1 \mathrm{mbps}$ \\
\hline Environment Size & $10 \times 10 \mathrm{Km}$ \\
\hline Traffic Type & Constant Bit Rate (CBR) \\
\hline Cluster Head Speed & $11 \mathrm{mbps}$ \\
\hline Packet Size & 1024 \\
\hline Network Protocol & $\mathbb{P}$ \\
\hline Transport Protocol & ICP \\
\hline Propagation .Model & Random /Tay Point .Yode \\
\hline MAC Protocol & 802.11 \\
\hline
\end{tabular}

Table 1: Parameters used for experimentation

\section{RESULTS}

This research focused on providing solution for said problem by implementing ant colony algorithm with clustering approach in OPNET simulator.

Research provides solution over fault-tolerance using antoptimization. Here in our research collectively implement four scenarios for simple AODV network, clustered network using AODV Routing protocol, simple ant colony optimization on clustered network and last one for ant-colony with failure links in the AODV clustered network.

The through put, delay, network load i.e. quality of service constrain parameters of the simple, clustered and simulation with ant colony method with and without failure in network is given below. 


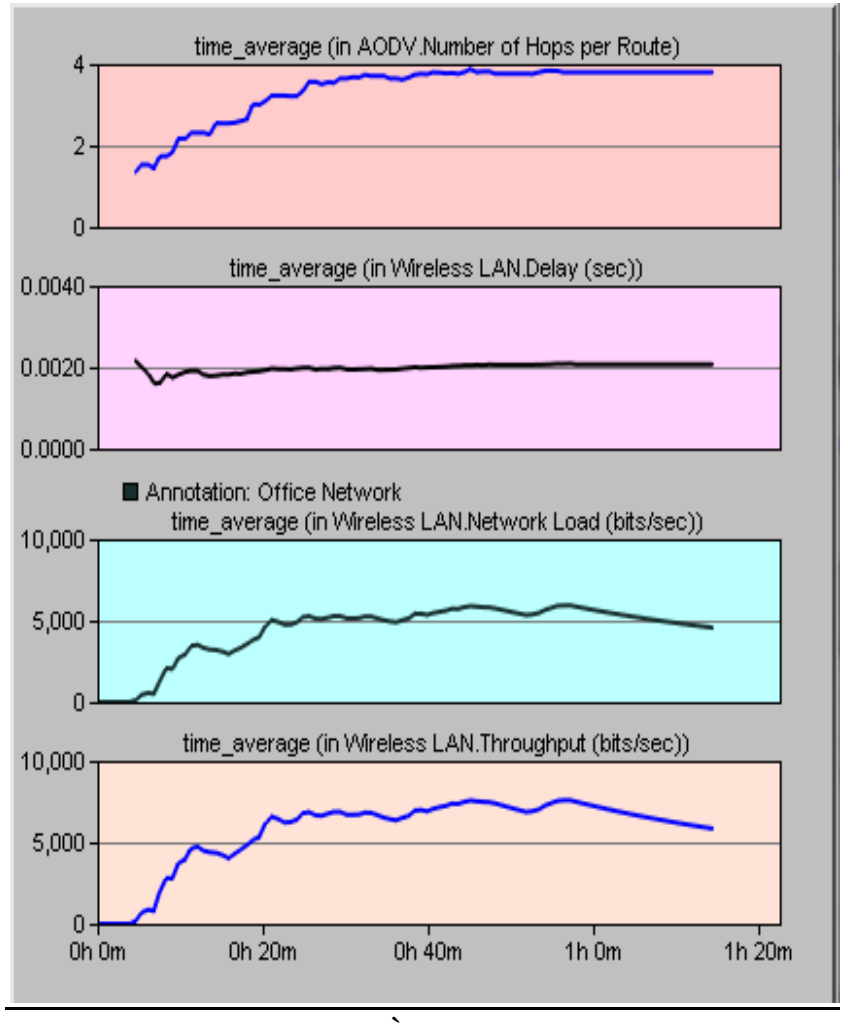

Figure 2: Result scenario with simple AODV

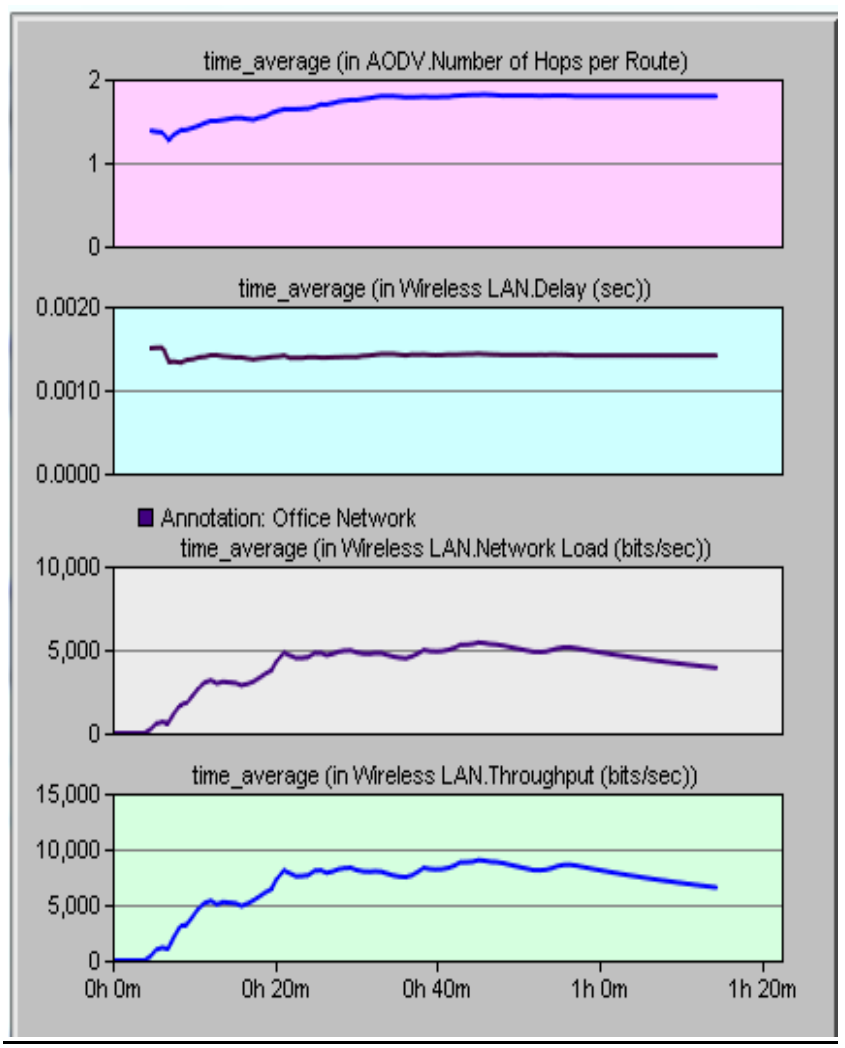

Figure 3: Result scenario with clustered network

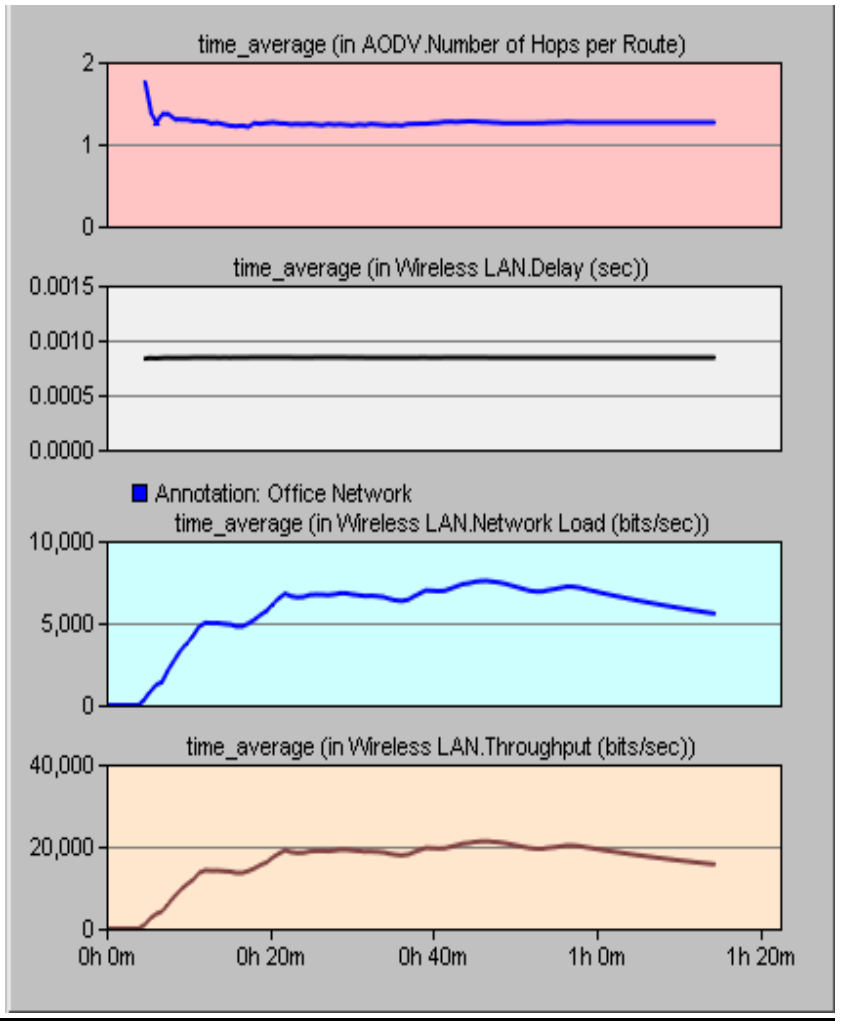

Figure 4: Result scenario with ant colony optimization on clustered network of AODV.

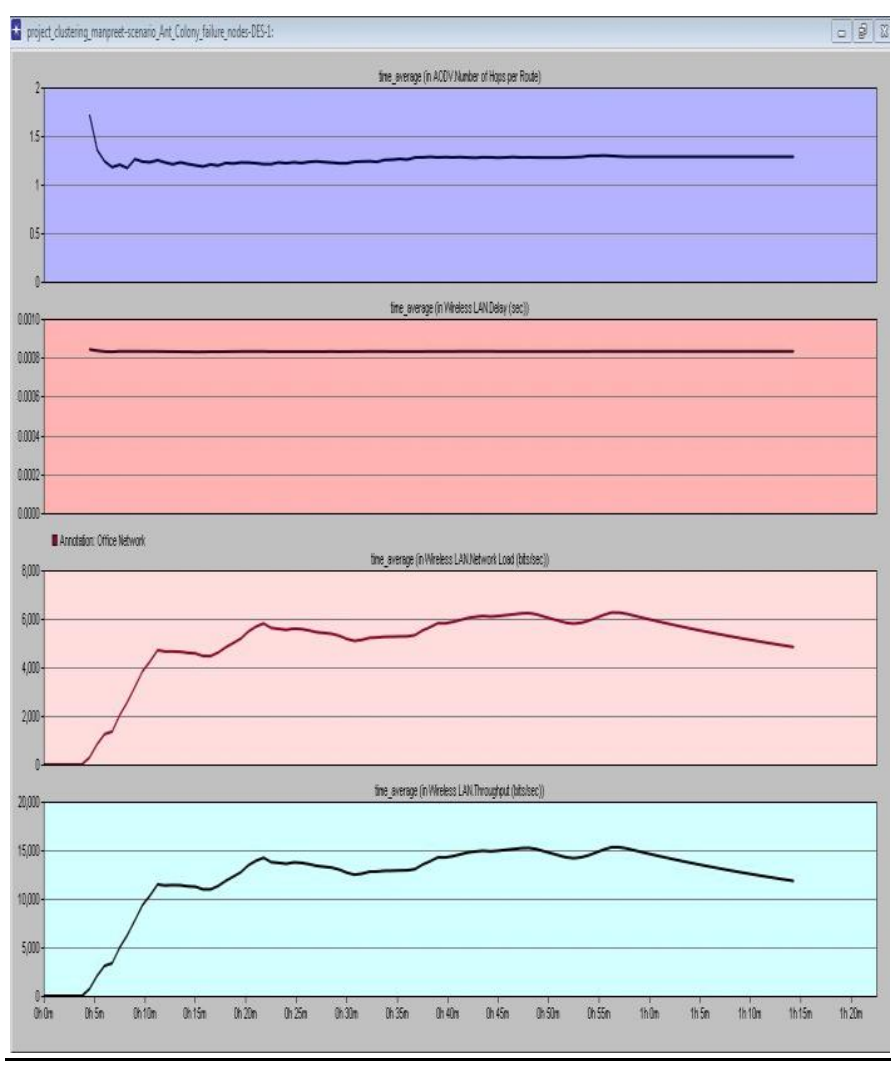

Figure 5: Result scenario with link failure within optimization technique in clustered network. 


\subsection{Comparative analysis of above four scenarios:}

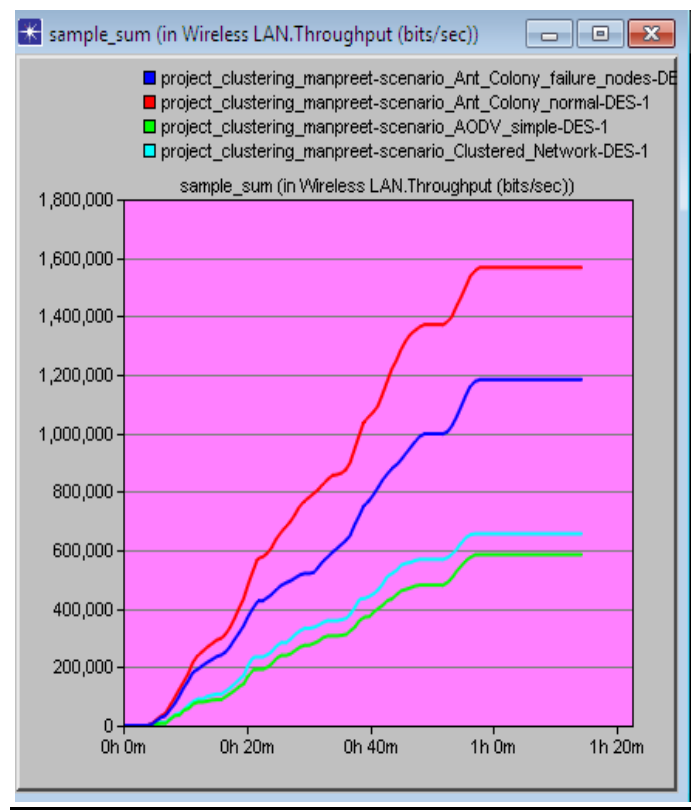

Figure 6: Throughput comparison of various simulations

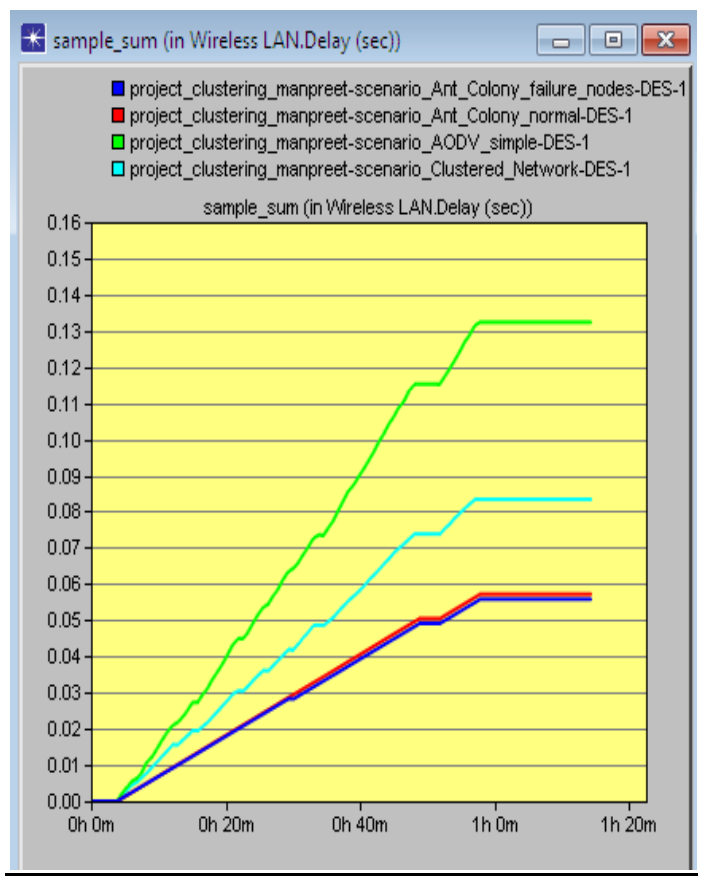

Figure 7: Delay comparison of various simulations

\section{CONCLUSION}

This presents a optimization technique using ACO algorithm that constructs always shortest path and improves QOS parameter with improving fault-tolerance network topology for wireless networks, because the fault-tolerant is the ability of a system to continue providing its specified service despite component failures. Research will prove to be a good solution for saving resources while finding the better fit clustering approach in Mobile Ad-hoc Network. Also provides us vision of clustering scheme in mobile ad-hoc network to increase throughput and decrease delay and in turns improve overall performance of the network.

In future optimization of clustering scheme can be done by other optimization algorithms such as Genetic and Bee colony algorithms.

\section{REFERENCES}

[1] C. Perkins and P. Bhagwat, "Highly dynamic destination sequenced distance vector routing (DSDV) for mobile computer," Computer Communication Review, October 1994.

[2] T. Clausen and P.Jacquet. "Optimized link state routing protocol," http://www.tools.ietf.org/html/rfc3626, Vol.2, No. 2, pp 265-269, April 2006.

[3] S. Guha and S. Khuller, "An Approximation algorithm for connected dominating sets," Algorithm, Vol. 20, Issue. 4, pp. 374-87, April 1998.

[4] Routing protocols and concepts, CCNA exploration companion guide. "Introduction to dynamic routing protocols". Chapter three, pp 148-177.

[5] Yang Qin, “A Fault-tolerance Cluster Head Based Routing Protocol for Ad Hoc Networks", IEEE International conference of Advances in Engineering \& Technology, Vol.1, No. 5, pp 337-341, November 2008.

[6] Sudip Misra, Sanjay K. Dhurandher, "Using Ant-Like Agents for Fault-Tolerant Routing in Mobile Ad-Hoc Networks", IEEE International Conference of Computer Science and Network, Vol. 1, Issue. 3, pp. 12-16, June 2009.

[7] Y. Xue and K. Nahrstedt, "Fault Tolerant Routing in Mobile Ad hoc Networks", Proc. IEEE WCNC, New Orleans, 2003, pp. 1174-1179.

[8] Van Phuong T., Ngo Trong Canh, Young-Koo Lee, Sungyoung Lee, Heejo Lee, " Transmission Time-based Mechanism to Detect Wormhole Attacks", IEEE Conference on Asia-Pacific Service Computing Conference, pp 172- 178, December 2007.

[9] Ma Hongwei, "The Study on Ad hoc Networks Security Strategy based on Routing Protocols", IEEE International Conference on Computer Science and Network Technology, Vol.1, No.4, pp 445-449, December 2011.

[10] E.A.Mary Anita, V.Thulasi Bai, E.L.Kiran Raj, B.Prabhu, "Defending against Worm Hole Attacks in Multicast Routing Protocols for Mobile Ad hoc Networks" IEEE International Conference on Information Theory and Aerospace \& Electronics Systems Technology, pp 1-5, March 2011.

[11] Macro Darigo and Thomas Stutzle "Ant colony optimization", A Bradford Book the MIT Press, Cambridge, Massachusetts London, England.

[12] C.K.TOH, "Ad-hoc mobile wireless networks protocols and systems", published by Dorling Kindersly (India) Pvt.Ltd. 